\title{
ANÁLISE DAS EXPORTAÇÕES DE BAIXA INTENSIDADE TECNOLÓGICA DA REGIÃO SUL DO BRASIL: UMA ANÁLISE EMPÍRICA
}

\author{
Nome \\ Daniel Arruda Coronel \\ Instituição/Afiliação \\ Universidade Federal de Santa Maria (UFSM). \\ País \\ Resumo da \\ Biografia \\ Doutor em Economia Aplicada pela Universidade Federal de Viçosa (UFV), \\ Professor Associado do Departamento de Economia e Relações Internacionais \\ (UFSM) e Bolsista de Produtividade do CNPq. \\ Nome \\ Murilo Sagrillo Pereira \\ Instituição/Afiliação \\ País \\ Resumo da \\ Biografia \\ Doutorando em Engenharia de Produção pelo Programa de Pós-Graduação em \\ Engenharia de Produção e acadêmico do curso de Bacharelado em Estatística, \\ ambos na Universidade Federal de Santa Maria (UFSM). É Tecnólogo em \\ Fabricação Mecânica pela UFSM (2013). Especialista em Estatística e Modelagem \\ Quantitativa pela UFSM (2019). Mestre em Engenharia de Produção pela UFSM \\ (2017). \\ Nome \\ Adriano Mendonça Souza 䲿 \\ Instituição/Afiliação \\ Universidade Federal de Santa Maria (UFSM). \\ País \\ Resumo da \\ Biografia \\ Doutor em Engenharia da Produção pela UFSC. Professor Titular da UFSM, com \\ atuação no Programa de Pós-Graduação em Engenharia da Produção. \\ Nome \\ Leonardo Sangoi Copetti \\ Instituição/Afiliação \\ País \\ Resumo da \\ Biografia \\ Mestre em Administração pela Universidade Federal de Santa Maria (UFSM). \\ Contato principal para correspondência.
}

\section{RESUMO}

O presente estudo tem como objetivo analisar a relação das exportações de baixa intensidade tecnológica dos estados da Região Sul do país: Rio Grande do Sul, Santa Catarina e Paraná. Neste sentido, utilizou-se as séries temporais de valores mensais das exportações dos anos de 1997 a 2018, coletados no site da Fundação do Centro de Estudos do Comércio Exterior (FUNCEX). Realizou-se análises estatística descritiva das séries em estudo e modelagens vetoriais autoregressivas e de correção de erros - Vetores Autorregressivos (VAR) e Vetores de Correção de Erro (VEC). Os resultados indicaram que todos os estados apresentam comportamento semelhante em relação às exportações. Constatou-se que existe uma influência direta entre as exportações do RS e SC, assim como entre PR e RS. Ainda, pode-se inferir que essa influência é de longa dependência (1,5 semestres).

Palavras-Chave: Exportações de Baixa Intensidade Tecnológica. Séries Temporais. Região Sul 


\section{Revista \\ UNEMAT de \\ Contabilidade}

v. 8, n. 16,2019

\section{INTRODUÇÃO}

O crescimento econômico pode ser esboçado e analisado por meio das seguintes variáveis: a) existência de uma relação positiva entre o crescimento da indústria e o crescimento do produto agregado, ou seja, quanto maior for a taxa de crescimento do setor industrial, maior será a do produto nacional; b) o crescimento do produto industrial e da produtividade industrial se relacionam positivamente. Neste sentido, constata-se uma relação de causalidade, pois, quanto maior for a taxa de crescimento da indústria, maior será a taxa de crescimento da produtividade; c) a longo prazo, o crescimento da economia não seria restrito pela oferta, mas pela sua demanda. Neste contexto, a restrição de demanda ao crescimento do produto em uma economia aberta seria o balanço de pagamentos (KALDOR, 1957, 1978).

Segundo Oreiro (2015, p. 154), “em uma economia que já realizou seu processo de industrialização ou sua revolução capitalista e se tornou um país de renda média, o crescimento de longo prazo é determinado pela demanda agregada".

Com base no arcabouço keynesiano/kaldoriano, pode-se inferir que as exportações influenciam nas taxas de crescimento dos países através dos multiplicadores, os quais ajustam a taxa de crescimento do investimento e do consumo. Por fim, os países que obtêm alta elasticidade-renda da demanda internacional, oriunda de suas exportações, têm um melhor crescimento econômico (LIBÂNEO; MORO; LONDE, 2014).

Não obstante a isso, um dos grandes desafios que o setor industrial enfrenta está relacionado ao processo de desindustrialização, o qual pode ser positivo caso ocorra um aumento na participação de produtos com maior intensidade tecnológica em detrimento da transferência para o exterior de atividades intensivas em mão de obra ou com menor valor adicionado, contudo, se esse processo for oriundo de uma reprimarização da pauta exportadora, é prejudicial à competitividade do setor, a qual tem forte relação com a "doença holandesa", que consiste numa falha de mercado (OREIRO; FEIJO, 2010).

De acordo com o Ministério do Desenvolvimento, Indústria e Comércio (MDIC, 2018), a Região Sul é responsável por aproximadamente 12,04\% das exportações dos produtos de alta intensidade tecnológica, por $23,1 \%$ dos de média-alta intensidade tecnológica, por $4,62 \%$ dos de média baixa, por $29,09 \%$ dos de baixa intensidade tecnológica e por 31,15\% dos produtos não industriais. 
Ainda nesta perspectiva, segundo o Instituto Brasileiro de Geografia e Estatísticas (IBGE, 2019), a Região Sul é a segunda região com maior participação no PIB, visto que, de 2007 a 2018, apresentou uma média de 16,25\%. Já a participação dos estados foi, respectivamente, de 6,05\% (Paraná), 6,20\% (Rio Grande do Sul) e 4,00\% (Santa Catarina).

Conforme o MDIC (2018), em 2018, as exportações da região totalizaram US\$ 50 bilhões ( $2^{\mathrm{a}}$ posição no ranking de exportação das regiões brasileiras, com uma participação de 20,84\% nas exportações do país). No que tange às importações, o valor total foi de US\$ 39 bilhões ( $2^{\mathrm{a}}$ posição nacional, com uma participação de 21,59\%).

Ainda segundo o MDIC (2018), em 2018, a China foi o principal destino das exportações da Região Sul, com um total de US\$ 14 bilhões, representando $28 \%$ das exportações totais, seguida pelos Estados Unidos, com US\$ 5,53 bilhões (11,06\%), e a Holanda, com US\$ 2,74 bilhões $(5,48 \%)$. Os principais produtos exportados e suas participações nas exportações totais da região foram sementes, frutos oleaginosos e grãos com US\$ 12,52 bilhões $(25,04 \%)$, carnes e miudezas com US\$ 6,79 bilhões $(13,58 \%)$ e embarcações e estruturas flutuantes com US\$4,49 bilhões $(8,98 \%)$.

Apesar de tão importante participação, ainda há uma lacuna a ser preenchida visando analisar com maior acuidade como alterações no valor das exportações dos setores de baixa intensidade tecnológica dos estados do Paraná (PR), Rio Grande do Sul (RS) e Santa Catarina (SC) se relacionam no curto e no longo prazo. Neste sentido, este trabalho se diferencia dos de Coronel (2013), Bresser-Pereira (2019), Morceiro (2019) e Gonçalves et al. (2019), os quais analisaram questões inerentes à produção, à competitividade e à dinâmica das exportações, mas para a economia brasileira como um todo, não considerando as peculiaridades regionais.

A partir das respostas a essas questões, será possível formular estratégias e ações visando aumentar a competitividade do setor, bem como políticas que efetivamente sejam eficazes para dinamizar as exportações de baixa intensidade tecnológica, a qual tem importância estratégica para o Produto Interno Bruto (PIB) dessas regiões.

O presente artigo está estruturado em quatro seções, além desta introdução. Na segunda seção, apresentam-se o referencial teórico, na terceira os procedimentos metodológicos; na seção seguinte, os resultados são discutidos e analisados e, por fim, apresentam-se as principais conclusões do trabalho.

\section{REFERENCIAL TEÓRICO}


O setor industrial é um indutor do crescimento econômico, visto que este gera encadeamentos produtivos, economias de escala e externalidades para outros setores. Esse transbordamento das atividades industriais para os demais setores está relacionado à absorção de produtos e commodities produzidos no setor agrícola e de mineração, além da contratação de diversos tipos de serviços (KALDOR, 1966).

Ademais, é relevante destacar, com acuidade, que a indústria seria o lócus em que ocorre a maior parte da inovação tecnológica, pois promove maior produtividade e crescimento econômico. Dessa forma, a taxa de crescimento econômico está associada ao tamanho do setor manufatureiro na economia. Assim, os países que possuíssem uma alta participação de emprego no setor industrial frente aos demais setores apresentariam maiores elevações no PIB (SQUEFF, 2012).

Para autores como Szirmai (2012), Acemoglu e Robinson (2012), Bresser-Pereira, Oreiro e Marconi (2016) e Reinert (2016), todos os países que se desenvolveram tiveram seu modelo de crescimento puxado pelo setor industrial, visto que a produtividade nesse setor é maior do que na agricultura; o setor industrial oferece mais oportunidades de economias de escala que o setor agrícola, e os efeitos de encadeamento e transbordamento são maiores do que na agricultura.

A economia brasileira iniciou tardiamente o seu processo de industrialização, o qual teve um novo impulso a partir do Governo Vargas, na década de 1930, quando tem início o Processo de Substituição de Importações (PSI ${ }^{1}$ ), que vai até o final da década de 1980 e que adotou as seguintes medidas: desvalorização cambial; taxas múltiplas de câmbio; crédito e subsídios; elevadas tarifas de importação e forte participação do Estado, através de infraestrutura, logística e do arcabouço legal e institucional (CORONEL; AZEVEDO; CAMPOS, 2014).

Da década de 1990 até meados de 2000, foram poucas as ações para fomentar a competitividade do setor industrial, visto que o foco, no Brasil e demais países latinoamericanos, era o controle das taxas de inflação e a busca pela estabilidade macroeconômica (PERES, 2006).

Ainda nessa perspectiva, a perda de competitividade do setor industrial brasileiro, ao longo dos anos 2000, começou a levantar debates e discussões sobre se a economia brasileira estava passando por um processo de desindustrialização, o qual pode ser entendido como a

\footnotetext{
${ }^{1}$ Para uma análise com maior acuidade sobre o PSI, ver Coronel (2013).
} 


\section{Revista \\ UNEMAT de \\ Contabilidade}

v. 8, n. 16,2019

redução persistente da participação do emprego industrial relativamente ao emprego total, bem como queda da participação do setor industrial no PIB (ROWTHORM; RAMASWAMY, 1999; OREIRO; FEIJO, 2010; BRESSER-PEREIRA, 2011, 2019).

Os principais efeitos do processo de desindustrialização em países em desenvolvimento como o Brasil são a) queda na renda dos trabalhadores; b) especialização regressiva, com retorno às vantagens comparativas baseadas em recursos naturais (Teoria das Vantagens Comparativas); c) tendência a desequilíbrios externos; d) quedas nas taxas de investimento; e) queda no Produto Interno Bruto (PIB); f) aumento da importação de produtos industriais, sendo que as causas para tal processo estão relacionadas a sobrevalorização cambial, abertura econômica, altas taxas de juros, infraestrutura inadequada e alto custo Brasil (CANO; 2012, 2014; BENJAMIM, 2015).

Para Bresser-Pereira, Oreiro e Marconi (2016), quando um país passa por um processo de desindustrialização, algumas medidas devem ser adotadas, tais como desvalorização cambial, política industrial ativa, maiores investimentos em infraestrutura e sofisticação produtiva, ou seja, uma maior diversificação da produção.

\section{ASPECTOS METODOLÓGICOS}

Os valores das exportações, em dólares, dos produtos de baixa intensidade tecnológica dos estados do Paraná, Rio Grande do Sul (RS) e Santa Catarina (SC) foram coletados no site da Fundação do Centro de Estudos do Comércio Exterior (FUNCEX, 2019). Os dados utilizados neste trabalho compreendem o período de janeiro de 1997 a dezembro de 2018, totalizando 264 observações.

Com relação aos dados empregados, também é relevante destacar que esses preços não

foram deflacionados, pois, conforme Wang e Tomek (2007) e Siqueira (2007), o deflacionamento incorpora uma tendência nos dados, logo não faz sentido incluir uma tendência antes da realização do teste de cointegração.

\subsection{Teste de raiz unitária}

Um processo estocástico é estacionário quando a sua média e a sua variância forem constantes ao longo do tempo e quando o valor da covariância entre dois períodos de tempo depende apenas da distância, do intervalo ou da defasagem entre os períodos de tempo, e não 


\section{Revista \\ UNEMAT de \\ Contabilidade}

v. 8, n. 16,2019

do próprio tempo em que a covariância é calculada. Em termos de notação matemática, as propriedades do processo estocástico estacionário podem ser representadas por: (Média) $E\left(Y_{t}\right)=\mu$, (Variância) $\operatorname{var}\left(Y_{t}\right)=E\left(Y_{t}-u\right)^{2}=\sigma^{2}$ e (Covariância) $\gamma_{k}=E\left[\left(Y_{t}-\mu\right)\left(T_{t+k}-\mu\right)\right]$ (BUENO, 2008).

Um processo estocástico com as propriedades descritas anteriormente é conhecido, na literatura de séries temporais, como processo fracamente estacionário, ou estacionário em covariância, ou estacionário de segunda ordem, ou estacionário em sentido amplo².

Para determinar a ordem de integração das variáveis de interesse utilizou-se o teste de raiz unitária de Dickey-Fuller Aumentado (ADF), que permite verificar a existência de raízes unitárias nas séries temporais, ou seja, se as variáveis são ou não estacionárias (DICKEY; FULLER, 1979, 1981).

O teste ADF consiste na estimação da seguinte equação por Mínimos Quadrados Ordinários e pode ser expresso, conforme Enders (1995), da seguinte forma:

$$
\Delta y_{t}=\alpha_{0}+\gamma y_{t-1}+\sum_{i=2}^{p} \beta_{i} \Delta y_{t-i+1}+\varepsilon_{t}
$$

$$
\text { com: } \gamma=-\left(1-\sum_{i=1}^{p} \alpha_{i}\right) \text { e } \beta_{i}=\sum_{j=i}^{p} \alpha_{j}
$$

em que: $\alpha_{0}$ é o intercepto; $\gamma$ descreve o comportamento da série temporal; $y$ representa a variável dependente; $\Delta$ é a representação do operador de diferença e $\varepsilon_{t}$ denota o erro, que se assume ser identicamente e independentemente distribuída. Para determinar o número de defasagens utilizadas no teste para eliminar a autocorrelação residual, utilizou-se o menor valor do critério de Schwarz (SBC).

O parâmetro de interesse nas regressões (sem intercepto e sem tendência; com intercepto; com intercepto e tendência) é $\gamma$, sendo que, se $\gamma=\mathbf{O}$, a série contém uma raiz unitária. Nesse teste, compara-se o resultado da estatística $\tau$ com os valores apropriados reportados por Dickey-Fuller para determinar se aceita ou se rejeita a hipótese nula $\gamma=0$. A hipótese nula será rejeitada se o valor calculado da estatística $\tau$ for maior do que o valor crítico de Dickey-Fuller, indicando que a série é estacionária; caso contrário, a série é não-estacionária.

\footnotetext{
${ }^{2}$ Um processo estocástico é fortemente estacionário quando todos os momentos de sua distribuição não variam ao longo do tempo.
} 
O teste Kwiatkowski, Phillips, Shmidt e Shin (KPSS) é um teste alternativo ao ADF, sendo definido pelo procedimento de um teste de não estacionariedade contra a hipótese nula de estacionariedade, conforme Greene (2008). Formalmente é expresso pela seguinte expressão:

$$
\begin{aligned}
& y_{t}=\alpha+\beta t+\gamma \sum_{i=1}^{t} z_{i}+\varepsilon_{t} \\
& y_{t}=\alpha+\beta_{t}+\gamma Z_{t}+\varepsilon_{t}
\end{aligned}
$$

tendo as seguintes hipóteses:

$$
\begin{aligned}
& H_{0}: \gamma=0, \text { série é estacionária } \\
& H_{a}: \gamma \neq 0, \text { série é não estacionária }
\end{aligned}
$$

\subsection{Teste de causalidade de Granger}

Para verificar em que sentido alterações no valor exportações dos bens de baixa intensidade tecnológica do PR, RS e SC são transmitidas, empregou-se o teste de causalidade, seguindo a metodologia proposta por Granger (1969), que busca verificar se a incorporação de valores passados de uma variável $X$ contribui com melhores previsões para a variável $Y$. Portanto, trata-se de um teste de precedência temporal e não de causalidade no sentido de uma relação de causa e efeito.

\subsection{Análise de cointegração}

Com o objetivo de identificar o possível relacionamento de longo prazo entre as variáveis, utilizou-se o teste de cointegração elaborado por Johansen (1988). Utilizou-se, também, o Modelo Vetorial de Correção de Erro (VEC) para analisar o relacionamento econômico, de curto e longo prazo.

Mesmo que variáveis individuais não sejam estacionárias, mas exista pelo menos uma combinação linear estacionária das variáveis, então pode-se afirmar que essas variáveis são cointegradas (GREENE, 2008), ou seja, pode-se verificar uma relação de equilíbrio de longo prazo entre elas, que pode ser estimada e analisada. Engle e Granger (1987) mostram que, se todas as séries de interesse possuem a mesma ordem de integração $I(d)$ e existir um vetor $\alpha$, com $\alpha \neq 0$, em que a combinação linear dessas variáveis seja de ordem $d-b$, 
$Z_{t}=\alpha^{\prime} X_{t} \sim I(d-b), b>0$, pode-se afirmar que $X_{t}$ é um vetor de variáveis cointegradas denotadas por $X_{t} \sim C I(d, b)$.

O procedimento de Johansen (1988) para verificação de cointegração entre séries de tempo considera que todas as variáveis são endógenas e sua utilização não é limitada pela existência de endogeneidade do regressor (relação causal no sentido da variável dependente para a variável explicativa). Esse procedimento utiliza Máxima Verossimilhança para estimar os vetores de cointegração e permite testar e estimar a presença de vários vetores e não só de um único vetor de cointegração.

De acordo com Harris (1995), definido um vetor $z_{t}$ de $n$ variáveis potencialmente endógenas, é possível especificar o seguinte processo gerador, e modelar $z_{t}$ como um Vetor Auto-regressivo (VAR) irrestrito com k defasagens de $z_{t}$ :

$$
z_{t}=A_{1} z_{t-1}+\ldots+A_{k} z_{t-k}+u_{t}
$$

em que: $z_{t}$ é um vetor $(n x 1), A_{i}$ é uma matriz de parâmetros $(n x n)$ e $u_{t} \sim I I D\left(\mu, \sigma^{2}\right)$.

Ainda conforme Harris (1995), a Equação (2) pode ser reparametrizada em termos de um modelo Vetorial de Correção de Erro (VEC) esboçado como:

$$
\Delta z_{t}=\Gamma_{1} \Delta z_{t-1}+\ldots+\Gamma_{k-1} \Delta z_{t-k+1}+\prod z_{t-k}+\varepsilon_{t}
$$

em que: $\Gamma_{\boldsymbol{i}}=-\left(\boldsymbol{I}-\boldsymbol{A}_{1}-\cdots-\boldsymbol{A}_{\boldsymbol{i}}\right),(\mathrm{i}=1, \ldots, \mathrm{k}-1)$ e $\Pi=-\left(\boldsymbol{I}-\boldsymbol{A}_{1}-\cdots \boldsymbol{A}_{\boldsymbol{k}}\right)$. Da forma como especificado, o sistema contém informações de curto e longo prazo a mudanças de $z_{t}$, via estimativas de $\hat{\Gamma}_{i}$ e $\hat{\boldsymbol{I}}$, respectivamente, onde $\Pi=\alpha \beta^{\prime}$, com $\alpha$ representando a velocidade de ajustamento ao desequilíbrio e $\beta$ a matriz de coeficientes de longo prazo.

A importância do modelo de correção de erros reside no fato de permitir a ligação entre aspectos relacionados à dinâmica de curto prazo com os de longo prazo (HAMILTON, 1994). Dessa forma, os mecanismos de correção de erros pretendem fornecer um caminho para combinar as vantagens de se modelar tanto em nível, que representa as relações de longo prazo, quanto em diferenças, que mostra as relações no curto prazo. Desta forma, tanto a dinâmica do processo de ajustamento de curto prazo quanto de longo prazo mão mantidas e ajustadas simultaneamente. 


\section{Contabilidade}

O número de vetores de cointegração depende do posto ou rank $(r)$ da matriz $\Pi$ I . Em termos de vetores de cointegração, têm-se três possibilidades, conforme Enders (1995): se o posto de $\Pi$ é completo, então as variáveis $Y_{t}$ são $\mathrm{I}(0)$, ou seja, significa que qualquer combinação linear entre as variáveis é estacionária e o ajuste do modelo deve ser efetuado com as variáveis em nível; se o posto de $\Pi$ é zero, então não há relacionamento de cointegração e o modelo deve ser ajustado com as variáveis em diferença e, quando $\Pi$ tem posto reduzido, há $r$ vetores de cointegração.

Para Enders (1995), o rank de uma matriz é igual ao número de raízes características estritamente diferentes de zero, que pode ser identificado por meio de dois testes estatísticos. O primeiro deles é o teste do traço, que testa a hipótese nula de que o número de vetores de cointegração distintos é menor ou igual a r contra a hipótese alternativa de que o número desses vetores é maior do que $r$.

Que pode ser definido por:

$$
\lambda_{\text {trace }}(r)=-T \sum_{i=r+1}^{n} \ln \left(1-\lambda_{i}^{\prime}\right)
$$

em que:

$\lambda_{i}^{\prime}=$ valores estimados das raízes características obtidos da matriz $\Pi ; T=$ número de observações.

O segundo teste é o do máximo autovalor, que testa a hipótese nula de que o número de vetores de cointegração é $r$ contra a hipótese alternativa de existência de $r+1$ vetores de cointegração, podendo ser expresso por:

$$
\lambda_{\max }(r, r+1)=-T \ln \left(1-\lambda_{r+1}^{\prime}\right)
$$

Verificada a cointegração entre as séries analisadas, estima-se o modelo VEC, conforme definido na expressão. O modelo empírico utilizado para a estimação das relações entre as exportações de bens de baixa intensidade tecnológica do Paraná, Rio Grande do Sul (RS) e Santa Catarina (SC) é dador por:

$$
\log \exp . P R=\alpha+\log \exp . R S+\log \exp . S C+\varepsilon_{t}
$$

Em que: 


\section{Contabilidade}

logexp.PR

= logaritmo das exportações de baixa intensidade tecnológica do Estado do Rio Grande do Sul (RS);

\section{logexp.RS}

$=$ logaritmo das exportações de baixa intensidade tecnoló gica do Estado do Paraná $(P R)$;

\section{logexp.SC}

= logaritmo das exportaçôes de baixa intensidade tecnológica do Estado de Santa Catarina (SC);e

$\varepsilon_{t=}$ vetor de perturbações

Além da estimação do modelo VEC, foram calculadas as funções impulso-respostas e a decomposição da variância para entender como uma variável pode ser utilizada para explicar o comportamento de outra.

\section{ANÁLISE E DISCUSSÃO DOS RESULTADOS}

\subsection{Análise descritiva}

A Figura 1 apresenta as três séries temporais logaritimizadas, cada uma com 264 observações, consideradas neste estudo.
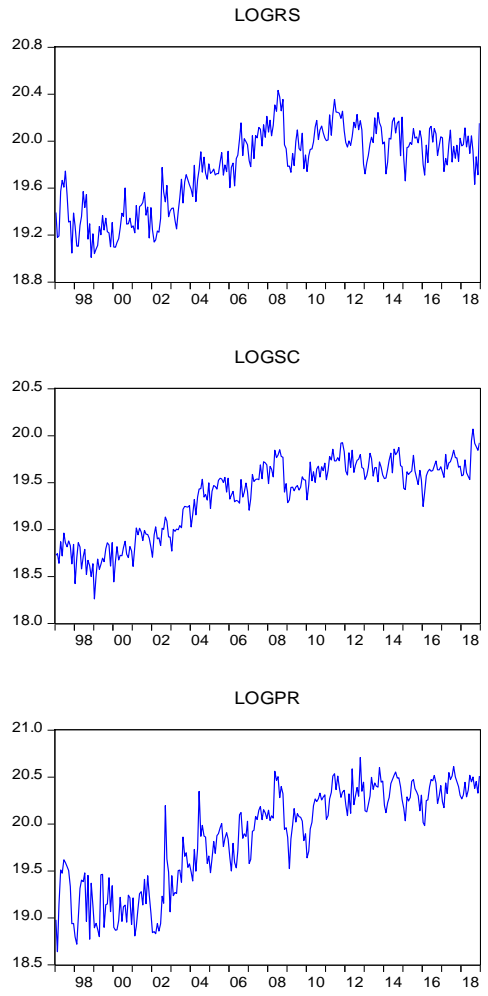
Figura 1 - Valor mensal das exportações de baixa intensidade tecnológica dos estados do Rio Grande do Sul, Santa Catarina e Paraná, de janeiro de 1997 a dezembro de 2018.

Fonte: Organização dos autores.

Por meio de análise da Figura 1, observa-se que as séries não são estacionárias e notase que as séries possuem comportamentos semelhantes no decorrer do tempo.

A Tabela 1 apresenta as medidas descritivas dos dados.

Tabela 1 - Medidas de estatística descritiva

\begin{tabular}{llcr}
\hline Medidas & logrs & logsc & logpr \\
\hline Média & 19,7721 & 19,3485 & 19,8580 \\
Mediana & 19,8519 & 19,4967 & 20,0176 \\
Máximo & 20,4329 & 20,0707 & 20,7095 \\
Mínimo & 19,0095 & 18,2604 & 18,6398 \\
Desvio padrão & 0,3402 & 0,4018 & 0,5357 \\
Coef. de variação (\%) & 1,7200 & 2,0770 & 2,6976 \\
Assimetria & $-0,4828$ & $-0,6181$ & $-0,5198$ \\
Curtose & 2,1613 & 2,1716 & 2,0217 \\
\hline
\end{tabular}

Fonte: Organização dos autores

Pela Tabela 1, observa-se que a série logpr apresentou a maior média e maior variabilidade dos dados, constatado pelo coeficiente de variação. Isso sugere uma maior heterogeneidade quando comparado às outras duas séries e a série mais homogênea corresponde ao estado do Rio Grande do Sul (RS). Vale ressaltar que, embora tenham sido elencadas as maiores médias e maiores variações dentre as séries, ambas se mostraram com comportamento muito semelhante, sendo um indício de que as séries sejam cointegradas.

\subsection{Teste de raiz unitária}

Conforme resultados apresentados na Tabela 2, constata-se que as séries são não estacionárias em nível, entretanto, após uma diferenciação, elas tornam-se estacionárias, sendo assim, integradas de ordem 1 - I(1).

Tabela 2 - Resultados dos testes ADF e KPS em nível para as séries mensais das exportações de baixa intensidade tecnológica dos estados do Rio Grande do Sul, Santa

Catarina e Paraná, de janeiro de 1997 a dezembro de 2018

\begin{tabular}{ccccc}
\hline \multirow{2}{*}{ Variáveis } & \multicolumn{3}{c}{ Nível } & \multicolumn{2}{c}{$1^{\text {a }}$ Diferença } \\
\cline { 2 - 5 } & ADF $^{\mathrm{a}}$ & $\mathrm{KPSS}^{\mathrm{b}}$ & ADF & KPSS \\
\hline $\log (\mathrm{RS})$ & $0,4901^{*}$ & $1,622^{*}$ & $<0,001^{*}$ & $0,0670^{*}$
\end{tabular}




\begin{tabular}{ccccc}
$\log (\mathrm{SC})$ & $0,6034^{*}$ & $1,828^{*}$ & $<0,001^{*}$ & $0,1398^{*}$ \\
$\log (\mathrm{PR})$ & $0,8534^{*}$ & $1,957^{*}$ & $<0,001^{*}$ & $0,0735^{*}$ \\
\hline
\end{tabular}

Fonte: Organização dos autores.

Nota: a: Teste Dickey-Fuller aumentado; b: Teste KPSS; d: Valores críticos com 5\% de significância; * modelo apenas com constante

Verificada a estacionariedade de primeira ordem, buscou-se analisar relação entre as variáveis que farão parte do modelo. As variáveis precisam estar relacionadas para que possam fazer parte do modelo.

\subsection{Teste de causalidade de Granger}

Conforme Tabela 3, observa-se que existe uma causalidade unidirecional da variável logrs com a variável logsc. Ainda, esse mesmo tipo de causalidade existe entre a variável logpr sobre a variável logrs. Dessa forma, verificado que existe relação entre as variáveis, buscou-se determinar o número de defasagens (ordem) para o modelo VAR. Esse modelo foi estimado sem constante, por esse ter apresentado menor BIC, quando comparado ao modelo com constante.

Tabela 3 - Resultados do Teste de Causalidade de Granger

\begin{tabular}{lll}
\hline Causalidade de Granger & Estatística F & p-valor $(<0,10)$ \\
\hline logrs causa logsc & 2,996 & 0,0510 \\
logpr causa logrs & 9,290 & 0,0001 \\
\hline
\end{tabular}

Fonte: Organização dos autores

A decisão do número de defasagens foi tomada conforme o número de defasagens que minimizou os Critérios de Informação de Schwarz (SC), de Akaike (AIC) e o de Hannan-Quinn $(H Q)$, procedimento semelhante ao que foi utilizado por Sousa, Amorim e Coronel (2012) e Silva, Coronel e Viera (2014). Os resultados de cada critérios estão expostos na Tabela 4.

Tabela 4 - Definição do número de defasagens do modelo VAR, para as séries de valor mensal das exportações de baixa intensidade tecnológica dos estados do Rio Grande do Sul, Santa Catarina e Paraná, de janeiro de 1997 a dezembro de 2018

\begin{tabular}{cccc}
\hline Lag & AIC & SC (BIC) & HQ \\
\hline 1 & $-3,311$ & $-3,186^{*}$ & $-3,260$ \\
2 & $-3,397$ & $-3,147$ & $-3,296$ \\
3 & $-3,488$ & $-3,113$ & $-3,337^{*}$ \\
4 & $-3,517$ & $-3,017$ & $-3,316$ \\
5 & $-3,514$ & $-2,889$ & $-3,263$ \\
6 & $-3,576$ & $-2,826$ & $-3,274$ \\
7 & $-3,593$ & $-2,718$ & $-3,241$
\end{tabular}


$\frac{8}{\text { Fonte: Organização dos autores }}$

$-2,670$ $-3,268$

Conforme Tabela 4, nota-se que o critério AIC indicou 8 defasagens para o modelo VAR. Em contrapartida, os critérios SC e HQ indicaram 1 e 3 defasagens, respectivamente. Levando em consideração o princípio da parcimônia entre explicação e interpretabilidade do modelo, optou-se por levar em consideração a indicação do critério SC.

Definido o número de defasagens, estimou-se o modelo VAR considerado. Esse modelo foi usado para realizar o teste de exogeneidade de blocos, visando definir a ordem que cada variável irá compor o modelo, o resultado do teste é apresentado na Tabela 5.

Tabela 5 - Resultado do Teste de Exogeneidade de Blocos

\begin{tabular}{lll}
\hline Variáveis & Qui-Quadrado & p-valor \\
\hline logpr & 14,701 & 0,0001 \\
logrs & 2,953 & 0,0857 \\
logsc & 0,681 & 0,4092 \\
\hline
\end{tabular}

Fonte: Organização dos autores

Com as informações completas para elaboração do modelo VAR auxiliar, ele foi definido com 1 defasagem, sem constante e com ordem de variáveis conforme Tabela 5. O modelo final é apresentado na Tabela 6 .

Tabela 6 - Estimação do modelo VAR

\begin{tabular}{cccc}
\hline Variáveis & $\Delta$ logpr & $\Delta$ logrs & $\Delta$ logsc \\
\hline D(logpr $(-1))$ & $-0,4025$ & 0,1634 & 0,0052 \\
& {$[6,377]$} & {$[3,8348]$} & {$[0,1320]$} \\
\hline D(logrs $(-1))$ & 0,0641 & $-0,4742$ & $-0,0967$ \\
& {$[0,7132]$} & {$[-7,8068]$} & {$[-1,7185]$} \\
\hline D(logsc $(-1))$ & 0,0882 & $-0,0288$ & $-0,3389$ \\
& {$[0,8254]$} & {$[-0,3990]$} & {$[-5,0585]$} \\
\hline
\end{tabular}

Nota: os valores entre colchetes referem-se à estatística t.

Fonte: Organização dos autores

Na seção seguinte, estima-se o modelo VEC.

3.4 Análise do Vetor de Correção de Erros (VEC) 
Após definido o modelo VAR auxiliar, onde se definiu a ordem das variáveis que irão compor o modelo e o número de defasagens a ser utilizadas, iniciou-se a elaboração do modelo VEC. Vale ressaltar que um dos pressupostos é que haja cointegração entre as variáveis e portanto realizou-se o teste de cointregração de Johansen (1998) baseado no modelo VAR auxiliar. O resultado do teste indicou, ao nível de 0,05 de significância, que existem pelo menos duas cointegrações. Dessa forma, o modelo VAR auxiliar pode ser transformado em um modelo VEC por meio da inclusão da correção de erros, apresentado na Tabela 7.

Tabela 7 - Estimação do VEC referente à variável valor das exportações de baixa intensidade tecnológica do Paraná, Rio Grande do Sul e Santa Catarina, de janeiro de 1997 a dezembro de 2018

\begin{tabular}{cccc}
\hline Variáveis & $\Delta$ logpr & $\Delta$ logrs & $\Delta$ logsc \\
\hline$\alpha$ & $-0,2886$ & $-0,1244$ & 0,0517 \\
& {$[-5,0702]$} & {$[-3,1458]$} & {$[, 3917]$} \\
\hline \multirow{2}{*}{$\operatorname{logpr}(-1)$} & $-0,2694$ & 0,2208 & $-0,0191$ \\
& {$[-4,0913]$} & {$[4,8224]$} & {$[-0,4447]$} \\
\hline \multirow{2}{*}{$\operatorname{logrs}(-1)$} & 0,1442 & $-0,4396$ & $-0,1107$ \\
\hline $\operatorname{logsc}(-1)$ & {$[1,6496]$} & {$[-7,2303]$} & {$[-1,9377]$} \\
& $-0,1823$ & $-0,1454$ & $-0,2919$ \\
$\mathrm{C}$ & {$[-1,5836]$} & {$[-1,8168]$} & {$[-3,8793]$} \\
\hline \multirow{2}{*}{$\mathrm{C}$} & 0,0091 & 0,0037 & 0,0059 \\
\hline
\end{tabular}

Nota: os valores entre colchetes referem-se à estatística t.

Fonte: Organização dos autores

A Tabela 8 apresenta a decomposição da variância para o estado do Paraná.

Tabela 8 - Decomposição da Variância para o valor mensal das exportações de baixa intensidade tecnológica do estado do Paraná, de janeiro de 1997 a dezembro de 2018

\begin{tabular}{cccc}
\hline Período & logpr & logrs & logsc \\
\hline 1 & 100,0000 & 0,0000 & 0,0000 \\
2 & 97,7935 & 0,1873 & 2,0192 \\
3 & 94,0848 & 0,1802 & 5,7350 \\
4 & 89,8907 & 0,1497 & 9,9596 \\
5 & 86,0106 & 0,1572 & 13,8322 \\
6 & 82,4873 & 0,1492 & 17,3635 \\
7 & 79,5014 & 0,1564 & 20,3422 \\
8 & 76,9333 & 0,1581 & 22,9085 \\
9 & 74,7676 & 0,1638 & 25,0686 \\
10 & 72,9145 & 0,1674 & 26,9181 \\
11 & 71,3313 & 0,1716 & 28,4971 \\
12 & 69,9629 & 0,1750 & 29,8621 \\
\hline \multicolumn{4}{c}{ Fonte: Organização dos autores }
\end{tabular}




\section{Contabilidade}

Conforme Tabela 8, a variação do primeiro período da série de exportações do estado do Paraná é explicada totalmente pelo próprio estado; no segundo período, 97,79\% da variação das exportações de baixa intensidade tecnológica é explicada pelo próprio estado, enquanto 0,18\% é explicada pelo estado do Rio Grande do Sul e 2,0 \% pelo estado de Santa Catarina. Esses resultados podem ser corroborados pelo trabalho de Schuh et al. (2017), bem como de Favaretto (2019) e Coronel (2020).

Convém ressaltar que a explicação obtida pelo estado do Rio Grande do Sul possui característica de decrescimento até o final do primeiro semestre. Após isso, a explicação dada pelo estado tem tendência de crescimento. Ademais, a contribuição do estado de Santa Catarina, na variabilidade do valor das exportações do Paraná, é crescente desde o primeiro período, tais resultados podem ser corroborados pelo trabalho de Teixeira, Coronel, Oreiro (2019).

Para análise do impulso de resposta, consideraram-se as relações mostradas na Tabela 3. Os resultados das análises são apresentados nas Figuras 2 e 3.

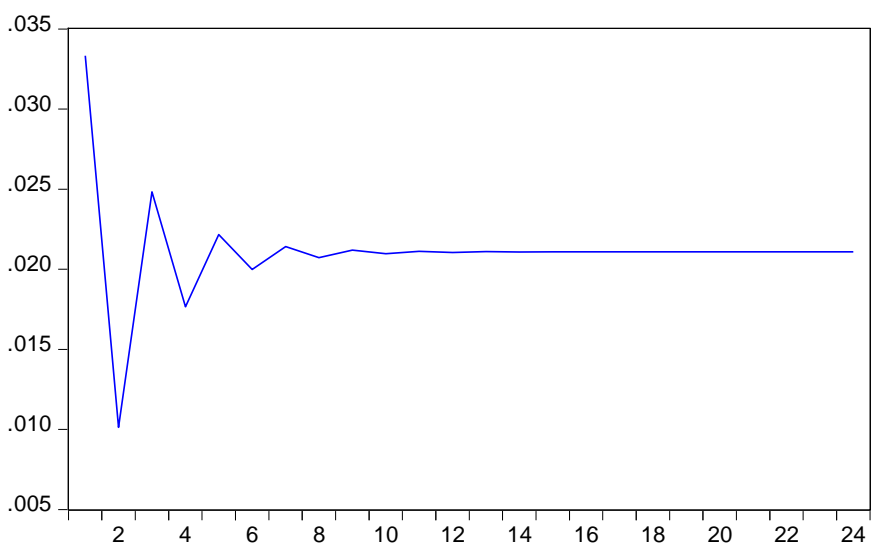

Figura 2 - Resposta de logsc em função de uma inovação em logrs usando fatores Cholesky Fonte: Organização dos autores

Conforme a Figura 2, pode-se verificar que um impulso em logrs ocasiona um efeito de redução na variável logsc, logo no primeiro período após o impulso. No período posterior, há aumento, entretanto não retorna ao patamar original. O impulso possui influência nos 9 períodos posteriores, após isso ocorre uma estabilização com valores relativamente moderados. 


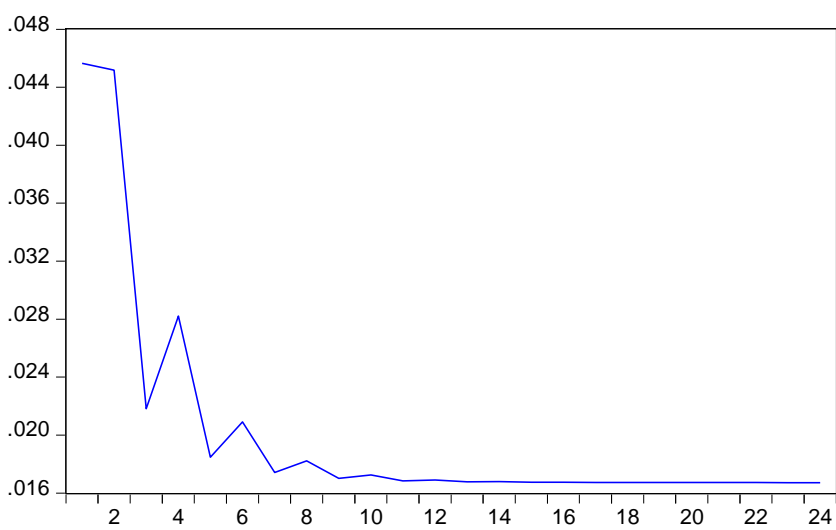

Figura 3 - Resposta de logrs em função de uma inovação em logpr usando fatores Cholesky Fonte: Organização dos autores

O impulso de logpr no logrs ocasiona uma redução mais acentuada do que a mostrada na Figura 2 e é possível verificar que, após aproximadamente nove meses, a série estabiliza. Faz-se pertinente ressaltar que a estabilização da série ocorre num valor muito inferior ao original. Ou seja, a influência do valor das exportações no estado do Paraná é grande e relativamente impactante no estado do Rio Grande do Sul. Esses resultados indicam a forte interdependência entre estes estados, os quais refletem-se na sua pauta exportadora.

\section{CONSIDERAÇÕES FINAIS}

O presente estudo permitiu identificar e analisar a relação entre os estados Rio Grande do Sul, Santa Catarina e Paraná, quando considerados os valores de exportações de baixa intensidade tecnológica. Isso foi permitido por meio de modelagem cointegrada das séries relativas a essas informações de cada estado, em um modelo VEC.

Como esperado, as exportações desse tipo apresentam uma tendência de crescimento significativa com o passar do tempo. Verificou-se também uma relação de causalidade entre os estados. As variações nos valores de exportação de baixa intensidade tecnológica do Rio Grande do Sul causam impacto nas exportações do mesmo tipo do estado de Santa Catarina, assim como o Paraná possui a mesma influência sobre o estado do Rio Grande do Sul. Essa influência foi constatada a longo prazo. Uma variação nas exportações do RS e PR ocasiona efeito de aproximadamente 1,5 semestres nas exportações de SC e RS, respectivamente.

Dessa forma, acredita-se que o desfecho deste estudo possa contribuir para uma melhor compreensão das relações existentes entre os estados da região Sul do país. Como limitações 
do presente trabalho, destaca-se a não inclusão de variáveis as quais impactem nas exportações destes estados, tais como taxa de câmbio e de juros, abertura comercial e renda externa, dentre outras.

Por fim, sugere-se, para trabalhos futuros, a replicação deste estudo para outros estados com a inclusão de variáveis macroeconômicas.

\begin{abstract}
The present study aims to analyze the relation of exports of low technological intensity of states, of South Region of the country, namely Rio Grande do Sul, Santa Catarina and Paraná. In this sense, we used temporal series of monthly values of these exports, during the period comprehended between the years of 1997 and 2018. The data were collected in the site of Foreign Trade Studies Centre Foundation (Fundação do Centro de Estudos do Comércio Exterior - FUNCEX). Descriptive statistics analysis of the temporal series was performed, as well as the modeling of the series via Vector Autoregressive Model (VAR) and Vector Error Correction Model (VECM). The results indicated that all the states presented similar behavior in relation to exports. We noted that there is a direct influence between the exports of RS and SC, as well as the ones between PR and RS. Yet, we can infer that this influence is of long dependence (1.5 semester).
\end{abstract}

Keywords: Exports of Low Technological Intensity; Temporal Series; South Region.

\title{
REFERÊNCIAS
}

BENJAMIN, C. Desindustrialização: pode o Brasil sobreviver sem um expressivo setor industrial? Boletim Conjuntura Brasil, Fundação João Mangabeira, n. 2, out. 2015.

ACEMOGLU, D.; ROBINSON, J. Por que as nações fracassam: as origens do poder, da prosperidade e da pobreza. Rio de Janeiro: Elsevier, 2012.

BRESSER-PEREIRA, L.C. Desenvolvimento, sofisticação produtiva, valor-trabalho e salários. Nova Economia (UFMG), v. 29, p. 135-160, 2019.

BUENO, R. D. L. D. Econometria de séries temporais. São Paulo: Cengage Learning, 2008.

CANO, W. A desindustrialização no Brasil. Economia e Sociedade, v. 21, Número Especial, p. 831-851, 2012.

CANO, W. (Des)Industrialização e (Sub)Desenvolvimento. Cadernos do desenvolvimento, v. 9, n. 15, p. 139-174, 2014.

CORONEL, D. A. Impactos da política de desenvolvimento produtivo na economia brasileira. Curitiba-PR-Brasil: Prismas, 2013. 
CORONEL, D.A. Análise do processo de desindustrialização na região Sul do Brasil: uma abordagem através de econometria de séries temporais. Monografia (Especialização em Estatística e Modelagem Quantitativa) - Universidade Federal de Santa Maria, 2020.

DICKEY, D. A.; FULLER, W. A. Distribution of the estimators for autoregressive time series with a unit root. Journal of the American Statistical Association, v.74, n.366, p.427-431, 1979.

DICKEY, D. A.; FULLER, W. A Likelihood ratio statistics for autoregressive time series with a unit root. Econometrica, v.49, p.1057-1073, 1981.

ENDERS, W. Applied Econometric Time Series. Nova York: John Wiley \& Sons, 1995.

ENGLE, R. F.; GRANGER, C. W. Co-integration and error-correction: representation, estimation and testing. Econometrica, Chicago, v.55, n.2, p. 251-276, 1987.

FAVARETTO, L. et. al. Influência da Taxa de Câmbio e Renda Externa sobre exportações gaúchas de produtos básicos (2001-2018). In: $\mathbf{2 3}^{\mathbf{0}}$ Congresso Brasileiro de Economia (CBE), Anais, Florianópolis, SC, 2019. Disponível em:

http://www.cbe2019.com.br/evento/cbe2019/trabalhosaprovados. Acesso em: 20 de agosto, 2019.

FUNDAÇÃO CENTRO DE ESTUDOS DO COMÉRCIO EXTERIOR (FUNCEX). Estatísticas. Disponível em: https://www.funcex.org.br. Disponível em: 18 de julho de 2019.

GONÇALVES, E. et al. Crescimento do emprego industrial local no Brasil: o grau de especialização por intensidade tecnológica importa. Nova Economia, v. 29, n. 1, p. 41-74, 2019.

GRANGER, C. W. J. Investigating causal relations by econometric models and cross spectral methods. Econometrica, Chicago, v. 37, n. 3, p.424-438, 1969.

GREENE, W. H. Econometrics Analysis. 6.ed. New Jersey: Pearson Education, 2008.

HAMILTON, J. D. Time series analysis. New Jersey: Princeton University Press, 1994.

HARRIS, R.I.D. Using cointegration analysis in econometric modelling. London: PrenticeHall-Harvester Wheatsheaf, 1995.

JOHANSEN, S. Statistical analysis of cointegration vectors. Journal of Economic Dynamic and Control, v.12,p.231-254,1988.

KALDOR, N. A model of economic growth. Economic Journal, v. 67, n. 268, p. 591-624, 1957.

KALDOR, N. Causes of the slow rate of economic growth of the United Kingdom. Cambridge: Cambridge University Press, 1966. 
KALDOR, N. Causes of the low rate of growth of the United Kingdom. Further Essays in Economic, Growth, London Duckworth, 1978.

LIBÂNIO, G.; MORO, S.; LONDE, A.C. Qualidade das exportações e crescimento econômico nos anos 2000. ENCONTRO NACIONAL DE ECONOMIA-ANPEC, 42. Anais... Natal, 2014.

MINISTÉRIO DO DESENVOLIMENTO DA INDÚSTRIA E COMERCIO EXTERIOR (MIDIC). Secretaria de Comércio Exterior (SECEX). Disponível em: <http://www.comexbrasil.gov.br/conteudo/ver/chave/secex/menu/211>. Acesso em: 31 ago. 2018.

MORCEIRO, P.C. Influência metodológica na desindustrialização brasileira e correções na composição setorial do PIB. Texto para Discussão, n.2. São Paulo: Nereus, 2019.

OREIRO, J.L. Um arcabouço teórico para a macroeconomia estruturalista do desenvolvimento: uma homenagem a Bresser-Pereira. In: OREIRO, J.L.; DE PAULA, L.F.DE.; MARCONI, N. A teoria econômica na obra de Bresser-Pereira. Santa Maria: Editora da UFSM, 2015.

OREIRO, J. L.; FEIJÓ, C. A. Desindustrialização: conceituação, causas, efeitos e o caso brasileiro. Revista de Economia Política, v.30, n.2, p.219-232, 2010.

REINERT, E. S. Como os países ricos ficaram ricos... e porque os países pobres continuam pobres. Rio de Janeiro: Contraponto, 2016.

ROWTHORN, R.; RAMASWAMY, R. Growth, trade and deindustrialization. Washington: International Monetary Fund Staff Papers, v. 46, n. 1, 1999.

SCHUH, A. B. et al. Perfil industrial do Rio Grande do Sul e a hipótese de desindustrialização. Perspectiva Econômica, v. 13, p. 116-133, 2017.

SILVA, F. M. da ; CORONEL, D. A. ; VIEIRA, K. M. . Causality and Cointegration Analysis between Macroeconomic Variables and the Bovespa. Plos One, v. 9, p. e89765, 2014.

SIQUEIRA, K. B. The dynamics of farm milk price formation in Brazil. Viçosa: UFV, Tese (Doutorado em Economia Aplicada) - Programa de Pós-Graduação em Economia Aplicada, Departamento de Economia Rural, Universidade Federal de Viçosa, Viçosa, 2007.

SOUSA, E. P ; AMORIM, A. L. ; CORONEL, D. A. . Abertura comercial e seus efeitos na transmissão de preços entre os mercados de trigo argentino e internacional. Revista de Economia (Curitiba), v. 37, p. 21-46, 2012.

SQUEFF, G. C. Desindustrialização: luzes e sombras no debate brasileiro. Brasília: IPEA, 2012. (Texto para discussão 1747).

SZIRMAI, A. Industrialisation as an engine of growth in developing countries, 1950-2005. Structural change and economic dynamics, v. 23, n. 4, p. 406-420, 2012. 
TEIXEIRA, F. O. ; CORONEL, D. A. ; OREIRO, J. L. . Principais determinantes do comportamento e da intensidade tecnológica das exportações brasileiras. In: XII Encontro Internacional da Associação Keynesiana Brasileira (AKB), 2019, Campinas-SP-Brasil. Anais do XII Encontro Internacional da Associação Keynesiana Brasileira (AKB), 2019. p. 0118.

WANG, D; TOMEK, W. G. Commodity prices and unit root tests. American Journal of Agricultural Economics, v. 89, n. 4, p. 873-889, 2007. 